Check for updates

\title{
Functional Outcomes of Revision Total Knee Arthroplasty Following Failed Unicompartmental Knee Arthroplasty
}

\author{
Chris Ironside, MBBS, B.App.Sc (physiotherapy)'; Simon Coffey, FRACS, FAOrthA'; Guy Eslick, PhD, FACE'; \\ Rami Sorial, FRACS, FAOrthA
}

\begin{abstract}
Introduction: Unicompartmental knee arthroplasty (UKA) can be used to treat medial compartment osteoarthritis of the knee. Some of these knees will eventually fail, and need to be revised. There is controversy about using UKA in younger patients as a definitive procedure or as a means to delay total knee arthroplasty (TKA) because the outcomes of subsequent revision surgery may be inferior to a primary TKA.

Methods: We retrospectively reviewed a series of 46 revision TKA patients following failed UKA (UKA revisions) using functional outcomes questionnaires and compared the results with a cohort of age and gender matched primary TKA patients. Our hypothesis was that UKA revision surgery would be inferior to primary TKA surgery.

Results: Data was collected on 33 knees after a mean follow-up period of five years. There was no significant difference in the Oxford Knee Score (33.7 vs 37.1, p =0.09) or the Western Ontario and MacMasters Universities Arthritis Index (WOMAC) (24.8 vs. 19.1, p = 0.22). A subgroup analysis demonstrated that UKAs, which fail early, are more likely to produce an inferior outcome following revision surgery than those that survive more than five years.

Discussion: We conclude that UKA can be used effectively in appropriately selected patients, as the functional outcome of their subsequent revision to TKA is not significantly inferior to a primary TKA.

Keywords: unicompartmental knee arthroplasty, revision knee arthroplasty
\end{abstract}

\section{Introduction}

Over the past three decades unicompartmental knee arthroplasty (UKA) has been used as a treatment for mono-

1 Sydney Medical School Nepean, 62 Derby St, Kingswood, NSW, 2747, Australia

2 Department of Orthopaedics, Nepean Hospital, Derby St, Penrith, NSW, 2750, Australia

3 Department of Surgery, Nepean Hospital, Derby St, Penrith, NSW, 2750, Australia

(C) 2015 Chris Ironside, Simon Coffey, Guy Eslick, Rami Sorial. All rights reserved DOI: 10.15438/rr.4.4.83 • ISSN 2331-2262 (print) • ISSN 2331-2270 (online)

For complete copyright and licensing information please refer to the end of this article. compartment arthritis of the knee. In the 1970's the cemented unicompartmental prostheses resembling modern devices emerged $[1,2]$ and with new prosthetic technology and strict patient selection criteria, UKA has been developed as a successful treatment specifically for osteoarthritis and osteonecrosis [3]. The early selection criteria set out by Kozinn and Scott in 1989 required the patient to be of low activity demand, over the age of sixty, weight less than $82 \mathrm{~kg}$ and with monocompartment disease. However, many studies have subsequently concluded that UKA can be used in patients under sixty years old [4-6] and in pa- 
tients up to $90 \mathrm{~kg}$ in weight [4] with outcomes comparable to the early selection criteria.

UKA has been shown to have better post-operative range of motion (ROM), less perioperative morbidity [7] and biomechanics closer to that of a normal knee [8] when compared to TKA. In a 15 -year prospective trial, Newman found that Bristol Knee Scores remained superior to TKA 15 years after surgery[9]. Furthermore, UKA has even been shown to be functionally comparable to a normal knee [10]. With this knowledge in mind, UKA is becoming more frequently used as a treatment for younger patients with the plan to revise the UKA to a TKA when failure occurs [11].

When the UKA does fail, revision to a TKA is superior than revision to another UKA $[12,13]$. Usually, the revision to TKA can be performed using a primary unconstrained prosthesis [4] and is considered a straightforward procedure although stems and augments may be required when there is significant peri-prosthetic bone loss [14].

Several studies have looked at the functional outcomes when a UKA is revised to a TKA. However, most are small case series and there is little consistency in outcome measures, comparators and follow-up periods. Many of the more recent series compare UKA revision with primary TKA, and these have found the functional result of UKA revision surgery to be inferior to that of primary TKA [13, 15-19]. Based on this result, Pearse et al. and Chou et al. conclude that UKA should not be used to delay TKA. The purpose of this study was to review the long-term functional outcomes of UKA revision surgery and compare this to a cohort of primary TKA patients, and use subgroup analyses to determine prognostic indicators. This will provide information on the success of UKA revision and thus the role of UKA in younger patients with medial monocompartment arthritis. Our hypothesis was that UKA revision surgery would be inferior to primary TKA surgery.

\section{Methods}

A retrospective, matched-pair, cohort design was used to evaluate the functional outcome and survivorship of TKA converted from failed UKA. Ethics approval was gained from the Nepean and Blue Mountains Local Health District human research and ethics committee (12/04 LNR/12/NEPEAN/9). Patients were identified from the surgical records of two orthopaedic surgeons for the period between 1997 and 2011. Patients were selected for inclusion if they had had a UKA revision following a UKA that had failed for any cause. Patients who had gone on to have further surgical procedures on the same knee were also in- cluded. Patients undergoing primary TKA were chosen as the control group, and were selected from the same surgeons' records and matched for surgeon, gender, time of surgery (within six weeks of corresponding UKA revision) and age (within ten years of corresponding UKA revision). Two control patients were chosen for each UKA revision. All patients were cross-referenced with the Australian Orthopedic Association National Joint Replacement Registry to determine if any further surgery (re-revisions) had been performed at another institution and to compare our cohort with the same knee replacement population across Australia.

Patients were mailed a package containing a small questionnaire to identify current body mass index, comorbidities and any further surgical procedures performed on their knee as well as the Oxford Knee Score (OKS) and Western Ontario and MacMasters Universities Arthritis Index (WOMAC). Patients were asked to return the questionnaires in the self-addressed envelope provided.

Subjects who returned their questionnaire were included in the final analysis. The medical records of these patients were analysed to identify the type of implant used, the components used in the revision surgery, survivorship of the original UKA and further surgical procedures.

The OKS and WOMAC were chosen because they are both self-reporting questionnaires. The OKS has been shown to have good correlation with the American Knee Society Score [20] and produces a score between zero and 48 with 48 being the best score.

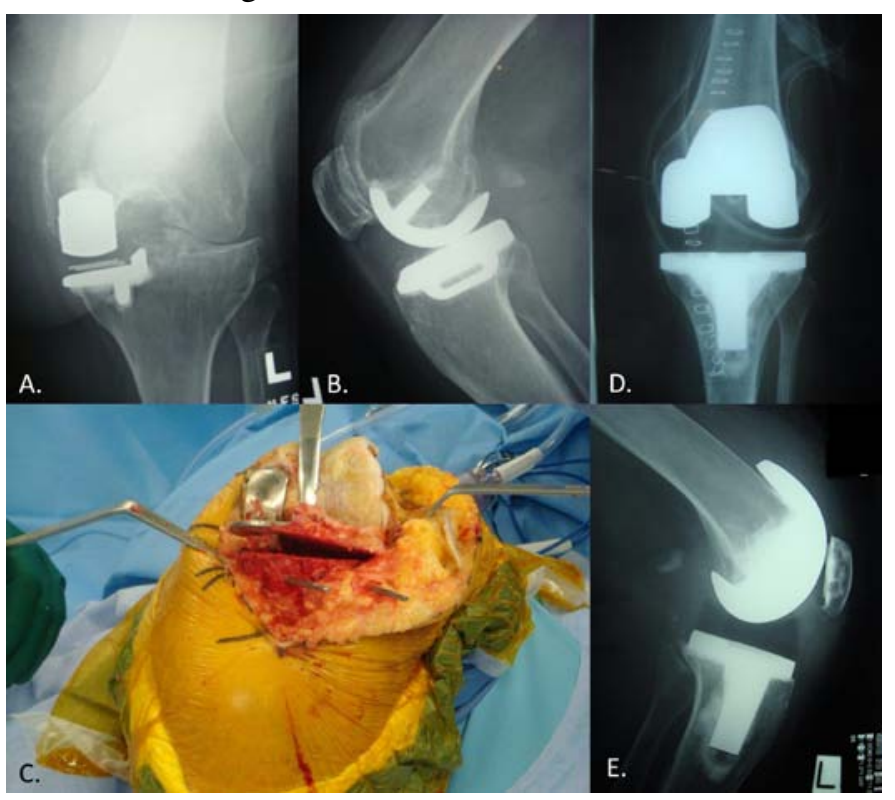

Fig 1. Failed cemented Oxford UKA at seven years. A) Pre-operative radiograph showing progression of disease to the lateral compartment. There is no loosening or collapse of the UKA. B) Sound fixation of the UKA from the lateral view. C) Intraoperative photograph of the tibia being prepared. Resection is performed at the level of the bone-implant interface to minimise bone loss and allow the revision component to be seated directly on host bone. D) and E) Post-operative radiograph showing good fixation. The larger bone resection on the tibia required a thicker polyethylene insert. 
The WOMAC is a validated questionnaire that has been used for many years as a disease specific instrument for hip and knee osteoarthritis [21]. The questions are divided into pain (five items), stiffness (two items) and function (17 items) categories. Using a five-point Likert scale the WOMAC produces a score between zero and 96 with zero being the best result.

Student's t-test was used to compare the means of each variable in the two groups. Kruskal-Wallis tests were used to compare the data of the subgroups within the UKA revision group. This test was chosen because of the small numbers in each group, differences in sample size between groups and because it was assumed there was an uneven distribution. The level of significance was set at 0.05 .

\section{Results}

Forty-three patients (46 knees) who had had UKA revision surgery were identified from the records of the authors. Ninety-two control patients (92 primary TKAs) were identified in the same way and matched to the study group. These patients were cross-referenced with the Registry of Births, Deaths and Marriages and 18 (six UKA revision patients) were found to be deceased. Thirty-one UKA revision patients (33 knees, 72\%) and 56 (61\%) control patients responded to the follow-up questionnaire. The mean follow-up time after revision surgery was 5.1 years (9 months to 14 years).

The commonest reason for failure of the UKAs requiring revision surgery was progression of disease (18) followed by loosening (12), ongoing pain (6), worn polyethylene (4), fractured tibial component (3), bearing subluxation (1), periprosthetic fracture (1) and recurrent haemarthrosis (1). The mean age of the original UKA was 61.5 and the mean time to failure was 5.7 years. There were no statistically significant differences in the baseline characteristics in the patients who responded to the questionnaire (table $1)$.

Table 1. Comparison of baseline biometric data between the two groups.

\begin{tabular}{|l|c|c|c|}
\hline & UKA $^{\dagger}$ revision & Primary TKA & P-value \\
\hline Number & 33 & 56 & \\
\hline $\begin{array}{l}\text { Mean age at } \\
\text { operation }\end{array}$ & 65.1 & 66.1 & 0.58 \\
\hline Gender (\%female) & 54.6 & 53.6 & 0.92 \\
\hline $\begin{array}{l}\text { Mean follow-up } \\
\text { years) }\end{array}$ & 5.1 & 4.2 & 0.12 \\
\hline Mean $\mathrm{BMI}^{\S}(\mathrm{kg} / \mathrm{m} 2)$ & 30.04 & 31.26 & 0.51 \\
\hline
\end{tabular}

† Unicompartmental Knee Arthroplasty

+ Total Knee Arthroplasty

$\S$ Body Mass Index
The OKS was not significantly different between the UKA revision and primary TKA groups (33.7 vs. 37.1 $\mathrm{p}=0.09$ ) after a mean follow-up period of 5.1 years. Similarly, the total WOMAC was not significantly different between the two groups for the same follow-up period ( 24.8 vs. $19.1 \mathrm{p}=0.22$ ). However, analgesia use was found to be significantly different with $27 \%$ of the UKA revision group using regular analgesia specifically for knee pain compared to only $5 \%$ of the primary total group $(\mathrm{p}=0.003)$ (table 2$)$.

Table 2. Outcome variables comparing the two groups.

\begin{tabular}{|l|c|c|c|}
\hline & UKA $^{\dagger}$ revision & Primary TKA & P-value \\
\hline Mean follow-up (years) & 5.1 & 4.2 & 0.59 \\
\hline OKS $^{\S}$ & 33.7 & 37.1 & 0.09 \\
\hline WOMAC" & 24.8 & 19.1 & 0.22 \\
\hline -Stiffness & 2.3 & 1.8 & 0.19 \\
\hline -Pain & 4.4 & 3.0 & 0.17 \\
\hline -Function & 18.1 & 14.6 & 0.25 \\
\hline Analgesia (\%) & 27.3 & 5.4 & 0.003 \\
\hline
\end{tabular}

+ Unicompartmental Knee Arthroplasty

$\$$ Total Knee Arthroplasty

\$ Oxford Knee Score

I/ Western Ontario and MacMasters Universities Arthritis index

The 33 UKA revision knees were further divided into three subgroups. Firstly, revisions from Oxford unicompartmental components $(n=17)$ were compared to revisions from all other unicompartmental components $(n=16)$ and no statistical difference in the outcome measures was found for either OKS (34.7 vs. $32.8 \mathrm{p}=0.65$ ) or WOMAC (21.4 vs. $28.0 \mathrm{p}=0.40)$. Likewise, there was no statistically significant difference found when revisions using primary total knee components $(n=22)$ were compared with revisions requiring stems or augments $(\mathrm{n}=11)$ for OKS (32.3 vs. $36.7 \mathrm{p}=0.46$ ) or WOMAC (30.3 vs. $13.8 \mathrm{p}=0.22$ ). There was, however, a statistically significant difference when revisions that were considered early failures (i.e.

Table 3. A comparison of UKA revision surgery for early and late failure.

\begin{tabular}{|l|c|c|c|}
\hline & $\begin{array}{c}\text { Revision for early } \\
\text { failure (<5yrs) }\end{array}$ & $\begin{array}{c}\text { Revision for late } \\
\text { failure (>5yrs) }\end{array}$ & P-value \\
\hline Number & 14 & 19 & 0.20 \\
\hline $\begin{array}{l}\text { Flexion ROM } \\
\text { (degrees) } \\
\text { months post-op }\end{array}$ & 109 & 116.9 & 0.003 \\
\hline OKS $^{\ddagger}$ & 26.7 & 39.1 & 0.001 \\
\hline WOMAC & 41.1 & 12.8 & $<0.001$ \\
\hline -stiffness & 4.0 & 1.6 & $<0.001$ \\
\hline -pain & 7.6 & 2.1 & 0.001 \\
\hline -function & 29.5 & 9.7 & 0.01 \\
\hline Analgesia (\%) & 50 & 11 & \\
\hline
\end{tabular}

$\dagger$ Range Of Motion

+ Oxford Knee Score

$\S$ Western Ontario and MacMasters Universities Arthritis index 
UKA failure earlier than five years) were compared to late failures. The early failure group had poorer results in both the OKS (26.7 vs. $39.1 \mathrm{p}=0.003)$ and WOMAC (41.1 vs $12.8 \mathrm{p}=0.001$ ) scores after a mean follow-up of five years as well as reporting higher rates of analgesia use (table 3 ).

Of the UKA revision patients there were two re-revisions. The first required a two-stage revision two months after revision surgery due to uncontrolled sepsis. The second re-revision was done 17 months following revision surgery in a patient who had ongoing pain and stiffness as a result of recurrent haemarthrosis while on Warfarin. In the control group one patient required revision 11 months after primary TKA and was revised with revision implants for ongoing symptoms.

\section{Discussion}

Our results show that after an average of five years follow-up there are no statistically significant differences in the function of patients following revision surgery for failed UKA patients compared to primary TKA patients as assessed by OKS and WOMAC scores. There is, however, a significant increase in analgesia use in the UKA revision cohort.

As UKA revision is a relatively uncommon procedure and there is a long time period between the original UKA and the follow-up after revision to TKA, the statistical power of this study is limited by sample size. It is possible that the lower functional scores in the UKA revision group is a true difference, but the sample size was not large enough to show statistical difference. The numbers in our study were comparable to other non-registry studies on the subject. Our study included UKA failure of any cause, including infection, as we wanted our results to reflect real life decision-making. There were however, no UKAs that failed due to infection.

The mean OKS in the UKA revision group was 33.7 (vs. 37.1 in the primary TKA group), which is slightly better than other studies of similar design. Pearse et al. [13] found a mean OKS of 30.02 (vs. 37.16 in the primary TKA group) and Chou et al. [16] found a mean OKS of 29 (vs. 39 in the primary TKA group). Both of these studies found that the index procedure was significantly poorer than primary TKA, and although the differences in our study were narrower, we concede that a greater sample size may have shown statistical significance. However, these studies have a shorter follow-up time of 6 months and 12 months, respectively. The study by Pearse et al. examined 122 patients through the New Zealand National Joint replacement registry and therefore looks at a large number of surgeons whilst Chou et al. looked at a series of 33 UKA revisions performed by eight surgeons. The two surgeons involved in our study are high volume arthroplasty surgeons, which may explain the higher outcomes scores.

We found a mean WOMAC score of 24.8 (vs. 19.1 in the primary TKA group [lower score indicates better result]), but again this was not a significant difference. This was similar to that found by Rancourt et al. [19] who reported a mean WOMAC score of 25.8 (vs. 19.8 in the primary TKA group) in their study of 63 UKA revisions after 3.1 years mean follow-up. It is also significantly better than Oduwole et al. [22], who found a mean WOMAC score of 33.3 in their series.

Several subgroup analyses were conducted on the data yielded from our study to help identify prognostic indicators. We found there was no significant difference in knees revised from an Oxford unicompartmental prosthesis compared with all other unicompartmental prostheses. The Oxford unicompartmental prosthesis has a mobile bearing on a keeled, polished tibial prosthesis. The other unicompartmental prostheses are a number of fixed bearing prostheses, which either have an all polyethylene tibial component or modular tibial components with small pegs for fixation. At the start of the study we had hypothesised that a prosthesis with a deeper tibial keel (such as the Oxford) may cause greater bone loss and hence result in a poorer revision. However, the results of our study demonstrate a slightly better result for revisions of the Oxford prosthesis (all except for one were cemented), although this was not significant. The keel of the Oxford tibial component leaves a small cavitary defect when performed well provided that it has not loosened or migrated. However, if done poorly or there is bone collapse has the potential to leave larger defects.

We also hypothesised that when a knee required revision components and/or grafts for revision it would also produce a poorer result than primary TKA components. Again, our results do not show a significant difference and even demonstrated a trend toward the contrary. This result might indicate that using revision components may actually produce a better outcome than trying to revise to primary implants at all costs, however, this would need to be examined in larger numbers. The general approach of the surgeons in this series was to use the least level of constraint required to produce a stable revision but given these results it is possible that a more stable prosthesis may have been indicated in some cases where only primary components were used.

Our final subgroup analysis showed a clear difference in revisions of unicompartmental knees that had failed early (within five years) and those that had failed later. Those 
that had failed later produced revisions with mean OKS and WOMAC scores similar to (or even better than) that of primary TKA.

Several recent studies have found that UKA revision is inferior to primary TKA and concluded that UKA should not be used as a conservative procedure to delay TKA [13, 16]. As the functional scores in our study were not significantly different between groups, we cannot concur with this conclusion. By using primary TKA as the control group we are able to compare UKA revision surgery to a well-known and successful procedure. It is also consistent with previous studies on this subject. However, it is still not an ideal comparison because it fails to recognise that joint disease has been occurring in the knees that initially received the UKA for a much longer period than those receiving a primary TKA. A slightly inferior result in the UKA revision group, as other studies have found [13,15-19], might be acceptable to surgeon and patient, as the original UKA has treated the symptoms of joint disease for many years.

Overall, we believe that UKA can be a definitive procedure and should not be treated as an interim solution. It can provide many years of symptomatic relief and in the majority of cases will not require revision. If a UKA does fail, our study shows that it can be converted to TKA with results that are equivalent to primary TKA but potentially 5 to 15 years after the original presentation.

\section{References}

1. Skolnick MD, Bryan RS, Peterson LF. Unicompartmental polycentric knee arthroplasty: description and preliminary results. Clinical Orthopaedics \& Related Research1975 Oct(112):208-14.

2. Insall J, Walker P. Unicondylar knee replacement. Clinical Orthopaedics \& Related Research1976 Oct(120):83-5.

3. Kozinn SC, Scott R. Unicondylar knee arthroplasty. Journal of Bone \& Joint Surgery - American Volume1989 Jan;71(1):145-50.

4. Berend KR, George J, Lombardi Jr AV. Unicompartmental knee arthroplasty to total knee arthroplasty conversion: Assuring a primary outcome. Orthopedics2009;32(9):684.

5. Kort NP, van Raay J, Cheung J, Jolink C, Deutman R. Analysis of Oxford medial unicompartmental knee replacement using the minimally invasive technique in patients aged 60 and above: an independent prospective series. Knee Surgery Sports Traumatology Arthroscopy2007 Nov;15(11):1331-4.
6. Swienckowski JJ, Pennington DW. Unicompartmental knee arthroplasty in patients sixty years of age or younger. The Journal of bone and joint surgery American volume2004;86 A Suppl 1(Pt 2):131-42.

7. Newman JH, Ackroyd CE, Shah NA. Unicompartmental or total knee replacement? Journal of Bone and Joint Surgery - Series B1998;80(5):862-5.

8. Hodge WA, Chandler HP. Unicompartmental knee replacement: a comparison of constrained and unconstrained designs. Journal of Bone \& Joint Surgery - American Volume1992 Jul;74(6):877-83.

9. Newman J, Pydisetty RV, Ackroyd C. Unicompartmental or total knee replacement THE 15-YEAR RESULTS OF A PROSPECTIVE RANDOMISED CONTROLLED TRIAL. Journal of Bone and Joint Surgery-British Volume2009 Jan;91B(1):52-7.

10. Willis-Owen CA, Brust K, Alsop H, Miraldo M, Cobb JP. Unicondylar knee arthroplasty in the UK National Health Service: an analysis of candidacy, outcome and cost efficacy. The Knee2009;16(6):473-8.

11. Bert JM. Unicompartmental knee replacement. Orthopedic Clinics of North America2005 Oct;36(4):513-+.

12. Lewold S, Robertsson O, Knutson K, Lidgren L. Revision of unicompartmental knee arthroplasty: outcome in 1,135 cases from the Swedish Knee Arthroplasty study. Acta Orthopaedica Scandinavica1998 Oct;69(5):469-74.

13. Pearse AJ, Hooper GJ, Rothwell A, Frampton C. Survival and functional outcome after revision of a unicompartmental to a total knee replacement: the New Zealand National Joint Registry. Journal of Bone \& Joint Surgery - British Volume2010 Apr;92(4):508-12.

14. Levine WN, Ozuna RM, Scott RD, Thornhill TS. Conversion of failed modern unicompartmental arthroplasty to total knee arthroplasty. Journal of Arthroplasty1996 Oct;11(7):797-801.

15. Becker R, John M, Neumann WH. Clinical outcomes in the revision of unicondylar arthoplasties to bicondylar arthroplasties. A matched-pair study. Archives of Orthopaedic and Trauma Surgery2004 Dec;124(10):702-7.

16. Chou DTS, Swamy GN, Lewis JR, Badhe NP. Revision of failed unicompartmental knee replacement to total knee replacement. Knee2011.

17. Jarvenpaa J, Kettunen J, Miettinen H, Kroger H. The clinical outcome of revision knee replacement after unicompartmental knee arthroplasty versus primary total knee arthroplasty: 8-17 years follow-up study of 49 patients. International Orthopaedics2010 Jun;34(5):649-53.

18. Miller M, Benjamin JB, Marson B, Hollstien S. The effect of implant constraint on results of conversion of unicomnartmental knee arthroplasty to total knee arthroplasty. Orthopedics2002 Dec;25(12):1353-7.

19. Rancourt M-F, Kemp KAR, Plamondon SMR, Kim PR, Dervin GF. Unicompartmental Knee Arthroplasties Revised to Total Knee Arthroplasties Compared With Primary Total Knee Arthroplasties. The Journal of arthroplasty2012;27(8, Supplement):106-10.

20. Reddy KIA, Johnston LR, Wang W, Abboud RJ. Does the Oxford Knee Score Complement, Concur, or Contradict the American Knee Society Score? The Journal of Arthroplasty2011;26(5):714-20.

21. Bellamy N, Buchanan WW, Goldsmith CH, Campbell J, Stitt LW. Validation study of WOMAC: a health status instrument for measuring clinically important patient relevant outcomes to antirheumatic drug therapy in patients with osteoarthritis of the hip or knee. J Rheumatol1988 Dec;15(12):1833-40.

22. Oduwole KO, Sayana MK, Onayemi F, McCarthy T, O'Byrne J. Analysis of revision procedures for failed unicondylar knee replacement. Irish Journal of Medical Science2010 Sep;179(3):361-4.

\section{Copyright \& Licensing}

Authors retain copyright and grant the journal right of first publication with the work. Reconstructive Review follows the Creative Commons Attribution-NonCommercial CC BY-NC. This license allows anyone to download works, build upon the material, and share them with others for non-commercial purposes as long as they credit the senior author, Reconstructive Review, and the Joint Implant Surgery \& Research Foundation (JISRF). An example credit would be: "Courtesy of (senior author's name), Reconstructive Review, JISRF, Chagrin Falls, Ohio". 